\title{
A structural investigation into an alpha-mannosidase found in Bacteroides thetaiotaomicron \\ Nicole Fraser ${ }^{1}$, David Rose ${ }^{2}$ \\ ${ }^{1}$ University of Waterloo ${ }^{2}$ Dept of Biology, Univ of Waterloo \\ n3fraser@uwaterloo.ca
}

Glycoside hydrolase family 38 (GH38) enzymes contain a myriad of alpha-mannosidases ranging in substrate specificity. All of the enzymes within this family share a common catalytic retaining mechanism with a conserved catalytic residue of aspartic acid. Despite structural studies across the kingdoms - notably from Drosophila melanogaster to Streptococcus pyrogenes - these enzymes vary strongly in kinetic preferences and structure. Recently, we've expressed a predicted alpha-mannosidase originating from Bacteroides thetaiotaomicron. This enzyme is predicted to contain a metal-binding region within the structure and may contain similar stabilizing disulfide-bridges similar to those in the Drosophila melanogaster alpha-mannosidase. The recombinant enzyme has been structurally modelled within ITASSER1,2,3 and current work focuses on crystallization of the enzyme for model validation.

1. Yang, J. Yan, R. Roy, A. Xu, D. Poisson, J. \& Zhang, Y. (2015). The I-TASSER Suite: Protein structure and function prediction. Nature Methods, 12, 7-8. doi:10.1038/nmeth.3213.

2. Roy, A. Kucukural, A. \& Zhang, Y. (2010). I-TASSER: a unified platform for automated protein structure and function prediction. Nature Protocols, 5, 725-738. doi: 10.1038/nprot.2010.5.

3. Zhang, Y.. (2008) I-TASSER server for protein 3D structure prediction. BMC Bioinformatics, 9(40). doi: 10.1186/1471-21059-40. 